\title{
Review Article \\ Coding and Noncoding Gene Expression Biomarkers in Mood Disorders and Schizophrenia
}

\author{
Firoza Mamdani, ${ }^{1}$ Maureen V. Martin, ${ }^{1}$ Todd Lencz, ${ }^{2,3,4}$ Brandi Rollins, ${ }^{1}$ \\ Delbert G. Robinson, ${ }^{2,3,4}$ Emily A. Moon, ${ }^{1}$ Anil K. Malhotra, ${ }^{2,3,4}$ and Marquis P. Vawter ${ }^{1}$ \\ ${ }^{1}$ Department of Psychiatry and Human Behavior, Functional Genomics Laboratory, University of California, \\ Irvine, CA 92697-4260, USA \\ ${ }^{2}$ Department of Psychiatry Research, Zucker Hillside Hospital, North Shore-Long Island Jewish Health System, 75-59 263rd Street, \\ Glen Oaks, NY 11004, USA \\ ${ }^{3}$ The Feinstein Institute for Medical Research, 350 Community Drive, Manhasset, NY 11030, USA \\ ${ }^{4}$ Department of Psychiatry and Behavioral Science, Albert Einstein College of Medicine of Yeshiva University, 1300 Morris Park Avenue, \\ Belfer Room 403, Bronx, NY 10461, USA
}

Correspondence should be addressed to Marquis P. Vawter; mvawter@uci.edu

Received 20 December 2012; Accepted 20 February 2013

Academic Editor: Daniel Martins-de-Souza

Copyright (C) 2013 Firoza Mamdani et al. This is an open access article distributed under the Creative Commons Attribution License, which permits unrestricted use, distribution, and reproduction in any medium, provided the original work is properly cited.

\begin{abstract}
Mood disorders and schizophrenia are common and complex disorders with consistent evidence of genetic and environmental influences on predisposition. It is generally believed that the consequences of disease, gene expression, and allelic heterogeneity may be partly the explanation for the variability observed in treatment response. Correspondingly, while effective treatments are available for some patients, approximately half of the patients fail to respond to current neuropsychiatric treatments. A number of peripheral gene expression studies have been conducted to understand these brain-based disorders and mechanisms of treatment response with the aim of identifying suitable biomarkers and perhaps subgroups of patients based upon molecular fingerprint. In this review, we summarize the results from blood-derived gene expression studies implemented with the aim of discovering biomarkers for treatment response and classification of disorders. We include data from a biomarker study conducted in firstepisode subjects with schizophrenia, where the results provide insight into possible individual biological differences that predict antipsychotic response. It is concluded that, while peripheral studies of expression are generating valuable results in pathways involving immune regulation and response, larger studies are required which hopefully will lead to robust biomarkers for treatment response and perhaps underlying variations relevant to these complex disorders.
\end{abstract}

\section{Introduction}

Psychiatric disorders affect a large percentage of the general population [1], and affected individuals present with mood alterations, psychosis, and in some cases combinations of both. Consequently, accurate diagnoses of mood and psychotic disorders may be deferred until certain criteria are met, resulting in treatment delays and decreased patient compliance with most patients undergoing multiple drug treatments. Given the plethora of treatment options available and the trial and error approach used in their administration, most patients do not respond favorably to treatment, and there is little data to predict individual treatment response. Whereas there is no "one size fits all" treatment course for the current diagnostic classifications, the possibility remains that, at an individual level, distinct medications may yield superior efficacy. Therefore, it is important to continue efforts to identify new ways to personalize treatment and improve outcomes. This approach may lead to a subgroup of patients, based upon molecular subtyping, that show better response to medications than other patients with a different molecular subtype. This review will focus on gene expression studies pertaining to treatment response in major depressive disorder (MDD), bipolar disorder (BD), and schizophrenia 
(SZ) and will provide an example of a biomarker study in individuals with first-episode SZ.

Pharmacogenetic studies have identified single nucleotide polymorphisms (SNPs) associated with treatment response to antipsychotics, mood stabilizers, and antidepressants (for reviews, see [2-5]). Serotonin and dopamine receptor polymorphism genotype, homovanillic acid levels, and severity of illness are a few of the predictive factors available, but the task of selecting the most effective treatment for each patient still remains a challenge $[3,6]$. Identification of novel early response biomarkers may enable patients to receive more individualized treatment, thereby reducing symptoms and adverse effects and increasing quality of life for patients. One area of genetic research that may in the future prove to be useful in classification of treatment and nontreatment responders will be the examination of methylation status of genomic DNA. This approach has been tried in peripheral samples in BD and SZ for demonstrating differences in methylation levels of single genes and genomewide between affected individuals and controls [7-10], while in MDD, a majority of methylation studies have been performed in postmortem brain $[11,12]$. In MDD, there have also been studies investigating the association of methylation and histone modifications with antidepressant treatment response (reviewed in [13]). For example, Lopez et al. [14] found that there is a significant decrease in H3K27me3 levels at promoter-IV of the BDNF gene in MDDs responding to 8 weeks of treatment with citalopram. BDNF is a gene of interest in depression since there is much evidence linking it to the etiology of depression, and its treatment [15]. Decreased BDNF gene expression has been associated with stress and depression and this downregulation can be reversed with antidepressant treatment [16].

The use of gene expression classifiers of antipsychotic, mood stabilizer, or antidepressant responses is an alternative strategy that may allow for prediction of individual drug response prior to or in the early phase of drug administration. Gene expression can change dynamically or remain state independent, as opposed to SNPs that are fixed and do not respond to medications or changes in psychiatric symptoms. Gene expression profiling has been successfully used in the investigation of response to treatment for several medical conditions, such as breast cancer [17-19], colon cancer [20], and cardiovascular disease [21-23]. These studies highlight the potential for the use of peripheral gene expression in investigations of the underlying pathophysiology of mood disorders and SZ. Although highly heritable, MDD, BD, and SZ are believed to be the product of multiple interacting genetic and environmental variables which can be investigated using gene expression [24]. The examination of gene expression alterations as potential biomarkers may allow for trait- as well as state-dependent markers of $\mathrm{BD}, \mathrm{MDD}$, and SZ subgroups, perhaps allowing insight into potential key biological processes implicated in these disorders.

\section{Peripheral Gene Expression}

The main interest in using peripheral blood for potential classifiers of antidepressant, antipsychotic, and mood stabilizer response, as opposed to brain tissue samples, is the relative ease of acquisition and the practical utility of blood samples. The peripheral blood transcriptome shares greater than $80 \%$ homology with genes expressed in the brain [25], heart, liver, spleen, colon, kidney, prostate, and stomach [26], as well as having its intensity of gene expression for a number of biological processes comparable to that of the prefrontal cortex [27].

The use of blood cells to perform microarray studies has become increasingly popular due to the numerous advantages it provides, including the possibility to collect larger sample sizes with a minimally invasive procedure [28]. The use of blood, from living subjects, to study gene expression avoids the influence of confounding variables associated with postmortem brain studies, such as the postmortem interval, low $\mathrm{pH}$, and other factors that decrease the integrity of mRNA and which must consequently be accounted for in subsequent analyses [29]. A recent gene expression study among psychiatric patients demonstrated the possibility of discriminating between schizophrenia and bipolar disorder using a blood-based protocol [30]. This same group has also confirmed findings implicating the selenium-binding protein 1 gene in schizophrenia using both brain and blood samples [31]. Recently, Rollins et al. [25] demonstrated considerable overlap between gene expression in brain and peripheral blood, from the same individual, using two independent populations and different high-throughput array platforms. A comprehensive study by Sullivan and colleagues [27] provides a pragmatic outlook on the use of peripheral gene expression and the comparability of results to that of the CNS. Sullivan et al. [27] carried out a secondary analysis on data stemming from 79 human tissues for 33,698 genes (probed using the Affymetrix U133A microarray). They observed that the nonparametric correlation between whole blood and CNS tissues was 0.5 and that only 21 genes from a group of $45 \mathrm{SZ}$ candidate genes were expressed in both the periphery and CNS and lastly that expression levels of genes in relevant biological processes were not significantly different between tissues. This study brings to attention, that although blood and brain are not $100 \%$ comparable, there are definite similarities in expression patterns which endorse the use of whole blood and its individual cells (lymphocytes, peripheral blood mononuclear cells) as a proxy for the brain, although some caution must be used in any interpretations made. Middleton and colleagues [32], when exploring lymphocyte gene expression in BD and SZ, observed opposing directionality of gene expression in lymphocytes to that of the same genes in the brain. The authors conclude, very importantly, that one should not disregard those findings present solely in the periphery or opposing findings made in the CNS due to their being of great potential in the use of peripheral blood in the study of psychiatric disorders. These reports are encouraging in that they provide positive evidence that peripheral markers are robust and are able to produce some findings that are comparable to those from postmortem studies, thus lending support to the use of peripheral blood samples as an advantageous alternative in the quest for the biological markers of brain-based disorders. 


\section{Noncoding RNA}

Only a few studies of noncoding RNA have been conducted for biomarkers in human subjects, while in preclinical investigations, there has been an explosion of noncoding RNA studies. An area of research related to noncoding RNA and gene expression is microRNA (miRNA). miRNA can control both the level and translation of mRNA [33], thereby coordinating spatial and temporal localization of gene expression and protein in tissue. Gardiner et al. [34] found miRNAs in the maternally expressed DLK1-DIO3 region on chromosome 14q32 to be downregulated in SZ peripheral blood mononuclear cells (PBMCs). Lai and colleagues [35] performed a miRNA study using a test set of $30 \mathrm{SZ}$ and 30 controls from which they identified a seven miRNA signature using supervised methods, and confirmed the discriminatory power of the signature in an independent cohort of $60 \mathrm{SZ}$, and 30 controls with an area under the curve of $85 \%$. miRNAs have not only been examined for phenotypic association, but also with regards to treatment response. Chen et al. [36] treated 20 lymphoblastoid cell lines (10 BD and 10 discordant siblings) with lithium and then analyzed the expression levels of 13 miRNAs. They observed significant changes in expression of seven miRNAs after four days of treatment and four of these (miR-34a, miR-152, miR-155, and miR-221) continued to exhibit expression changes at day 16 . Using miRanda and TargetScan to focus on the mRNA targets of miR-221 and miR-34a (previously found by [37] to have altered expression in rat hippocampus with lithium), they found 39 targets to be inversely correlated to miR expression. Several studies have recently been carried out to probe the relationship between miRNAs and antidepressant treatment and response. Bocchio-Chiavetto and colleagues [38] found 30 miRNAs to be differentially expressed in blood samples from ten depressed individuals following twelve weeks of antidepressant treatment with escitalopram. Oved et al. [39] profiled miRNAs, using microarrays, in eight lymphoblastoid cell lines, exhibiting high or low sensitivity to paroxetine. A comparison of these groups identified several miRNAs with significant expression differences between groups, particularly, miR-151-3p which targets CHL1, a gene implicated in neuronal plasticity. The serotonin transporter (SERT), which is the direct target of the SSRI class of antidepressants, was found to be the target of miR-16 [40]. In their study, Baudry et al. [40] determined that chronic treatment of mice with fluoxetine increased levels of miR-16 in the dorsal raphe thereby reducing SERT expression, similar to the function of SSRIs. These findings demonstrate a likely role of miRNAs in treatment response.

\section{Peripheral Gene Expression Studies for Identification of Diagnosis}

4.1. Major Depressive Disorder. Until recently, few microarray studies employing peripheral blood samples to investigate gene expression in MDD were available; the majority of studies used quantitative real-time PCR (qPCR). qPCR studies of blood gene expression have revealed a number of interesting alterations in MDD patients, including a reduction of glucocorticoid receptor alpha expression [41], reduction of glyoxalase-1 mRNA levels [42], reduced expression of neurotrophic factors [43], reduced PDLIM5 gene expression [44], and increased levels of CREB and HDAC5 [45]. The level of expression of the serotonin transporter in MDD patients is a source of inconsistency, with two studies showing increased levels $[46,47]$ in leukocytes and PBMCs and another study reporting decreased levels in lymphocytes [48].

Several studies exploring peripheral gene expression using microarray technology were recently published. Segman et al. [49] identified a gene expression signature capable of differentiating mothers prone to postpartum depression soon after childbirth. They observed a reduction in expression of genes involved in transcriptional activation, cell proliferation, immune response, and DNA replication and repair. Spijker et al. [50] investigated peripheral gene expression in MDD patients through stimulation and incubation of blood samples from MDDs and controls with lipopolysaccharide (LPS), a lipoglycan shown to produce depressive-like behaviors in humans [51], prior to RNA extraction. In this study, the authors found that there is a difference in LPSstimulated gene expression in MDD patients versus controls, leading to the identification of a predominantly immunerelated biomarker with $87.5 \%$ sensitivity and $61.5 \%$ specificity to differentiate between cases and controls.

4.2. Schizophrenia. Gene expression profiling using microarrays in peripheral samples from $S Z$ and controls has identified several likely candidates for future investigation [28]. Tsuang and colleagues [30] determined the utility of peripheral gene expression in the identification of a gene signature capable of discriminating between SZ, bipolar disorder, and controls. Their signature was composed of eight genes-APOBEC3B, ADSS, ATM, CLC, CTBP1, DATF1, CXCL9, and S100A9. This gene signature was derived using a cohort of $30 \mathrm{SZ}$, $16 \mathrm{BD}$, and 28 controls. Kurian et al. [52] identified 50 candidate biomarkers for SZ and psychotic disorders and 107 biomarkers for delusions from a sample of 31 subjects with SZ and related disorders. In a sample of 52 unmedicated SZ and 28 controls, Takahashi et al. [53] identified eight genes and two expressed sequence tags, expressed both in blood and brain, to differentiate between SZ and controls. Bowden et al. [54] identified altered peripheral gene expression in 18 genes with brain-associated functionality in a sample of $14 \mathrm{SZ}$ and 14 psychiatrically normal controls. A comparison of untreated first-episode schizophrenics $(N=32)$ to age- and gendermatched controls $(N=32)$ resulted in the identification of 180 probesets displaying significant differential expression between groups [55]. Maschietto et al. [56] identified an SZ classifier comprised of six genes (HERPUD1, HOXA13, CTNNA1, SULT1A1, PIK3R3, and MALAT1) able to discern between $\mathrm{SZ}$ and controls regardless of treatment.

4.3. Bipolar Disorder. Tsuang and colleagues [30], using blood-based gene expression, identified an eight-gene putative biomarker capable of discerning individuals with $\mathrm{BD}, \mathrm{SZ}$, and controls with $95 \%$ accuracy. Middleton et al. [32] demonstrated that peripheral gene expression can be successfully 
used to identify differences between affected individuals and their unaffected siblings in a sample of $33 \mathrm{SZ}$ and $5 \mathrm{BD}$ discordant sib-pairs.

The peripheral gene expression studies reviewed in this section demonstrate the feasibility of peripherally extracted coding and noncoding RNA to provide insights into brainbased disorders. One can reliably say that this area of research is valuable and continues to grow, although more studies are required to establish robust trait and response biomarkers. Those studies appear to be in progress; meanwhile, the utility of using blood as a neural probe continues to be frequently raised as an objection by critics to these studies. Certainly, if only single genetic causes of SZ, BD, or MDD were found and that RNA was only expressed in the brain, this argument would be convincing and valid. However, others in the field of molecular psychiatry would see neuroimmune markers influencing and interacting in both peripheral and central compartments and that these putative biomarkers could be relevant for the predisposition, state, and progression of these disorders.

\section{Peripheral Gene Expression of Treatment Response}

There has been limited, albeit promising, work published to date probing gene expression changes associated with treatment response in individuals with psychiatric disorders.

5.1. Major Depressive Disorder. Iga et al. [45] measured peripheral gene expression before and after treatment and reported high levels of histone deacetylase 5 (HDAC5) and cyclic-AMP response element binding protein 1 (CREB1) prior to treatment, with a significant reduction following 8 weeks of antidepressant treatment. Their findings for HDAC5 were recently confirmed by Hobara et al. [57] in a comparison of levels of HDAC5 in subjects with current MDD compared to a sample of MDD subjects in remission. A similar scenario was seen for the vascular endothelial growth factor (VEGF) gene, where mRNA levels were increased in the depressive state and diminished following antidepressant treatment. This decrease in VEGF mRNA levels, however, does not appear to translate into alterations in protein serum levels [58], suggesting the possibility of posttranscriptional modifications altering VEGF protein levels. However, the sample size used in this study was quite small $(N=25)$; thus, replication in larger treated samples is necessary.

Belzeaux et al. [59] identified specific candidate gene expression changes associated with antidepressant treatment response by qPCR; these included downregulation of the HDAC5 gene (confirming findings from studies outlined previously) and upregulation of serotonin receptors $1 B$ and 2A, the serotonin transporter, and CREB1 (which is in disagreement with previous findings) [45]. A limitation of this study was the impossibility of isolating gene expression changes specific to individual antidepressants, since the subjects in the study had undergone a variety of treatments. In a sample of $16 \mathrm{MDDs}$ and 13 matched control subjects, longitudinally followed for 8 weeks, Belzeaux and colleagues
[60] ascertained mRNA and miRNA differences between responders, nonresponders, and controls. The putative combination of four genes expression patterns (PPT1, TNF, IL1B, and HIST1H1E) could predict treatment response. In another longitudinal study investigating peripheral gene expression patterns of response to citalopram in a sample of 63 MDDs, Mamdani et al. [61] found interferon regulatory factor 7 (IRF7) to be the most significantly differentially expressed gene, with expression being upregulated in responders after 8 weeks of treatment. Furthermore, the IRF7 gene was found to exhibit decreased expression in the prefrontal cortex of subjects who died during a current depressive episode and were unmedicated, compared to controls.

5.2. Schizophrenia. Peripheral gene expression studies of antipsychotic response, as in the case of antidepressant response studies, are not numerous; however, they are encouraging while indicating a need for larger studies to be performed. The effect of treatment on gene expression compared to baseline/untreated levels was explored by Kuzman and colleagues [55] when 14 of their original 32 subjects achieved remission with second-generation antipsychotic treatment and were found to have control levels of DAAM2 compared to an increase prior to treatment initiation. VikMo and colleagues [62] determined that the expressions of fatty acid synthase and stearoyl-CoA desaturase were increased in treated individuals using a total sample of 38 psychotic subjects (19 treated with olanzapine monotherapy and 19 unmedicated). This result is not only an indication of treatment response; it might also be related to a weight gain drug effect observed with some antipsychotics. De and colleagues [63] performed a large peripheral gene expression study encompassing actively medicated SZ subjects $(N=$ 92), unmedicated SZ $(N=29)$, and 118 healthy controls. The authors focused on determining coexpression networks associated with SZ, regardless of treatment, in which they found that the most significant network branched out from the ABCF1 gene, a gene regulated by the major histocompatibility complex, and located in an SZ-associated genetic region $[64,65]$.

5.3. Bipolar Disorder. In a sample of 59 patients, with SZ or $\mathrm{BD}$ and having experienced their first psychotic episode, Gutiérrez-Fernández and colleagues [66] investigated the peripheral gene expression of CNPase $\left(2^{\prime}, 3^{\prime}\right.$-cyclic nucleotide $3^{\prime}$-phosphodiesterase) and MBP (myelin basic protein), prior to treatment initiation and after one year of treatment, and found no differences in gene expression between the two time points. Thus, these two genes do not appear to be possible treatment response biomarkers [66]. Beech et al. [67], in a group of 20 depressed BD patients and 15 controls, identified 1,180 genes having differential expression between cases and controls. However, there was no significant association of these 1,180 genes with response in a subgroup analysis of their initial $\mathrm{BD}$ cohort $(N=11$ treated with antipsychotics, $N=9$ untreated). In their study, Zain et al. [68] focused their inquiry on the antipsychotic olanzapine and the gene expression of PDLIM5 in BD subjects. They observed 
a significant decrease in symptom severity after 8 weeks of olanzapine treatment; however, the observed amelioration of symptoms was not correlated with gene expression. Further, they witnessed no significant differential expression between pretreatment and 4-week or 8-week treatment measures.

Kikuchi et al. [69] identified the gene expression of VEGFA (vascular endothelial growth factor A) to be downregulated by lithium in $\mathrm{BD}$ subjects using whole blood RNA and qRT-PCR; this observation corroborates previous findings of reduced VEGFA expression in lymphoblastoid cell lines treated with lithium [70]. These studies focused on the drug effects of lithium on expression, while Lowthert and collaborators (2012) [71] investigated gene expression associated to lithium response by performing a peripheral gene expression study with 20 depressed BD patients treated with lithium for 8 weeks. They observed 127 genes to show differential expression between response groups with apoptosis regulatory genes being upregulated in responders to lithium.

Collectively, studies of treatment response and antipsychotics on peripheral gene expression did not display significant effect although the drugs were effective in a subgroup of patients. This situation was different in the case of lithium, where there appeared to be at least some positive peripheral gene expression biomarkers of $\mathrm{BD}$ and lithium response, although they are not numerous; the studies were positive and invited future biomarker studies.

\section{Example of an Antipsychotic Biomarker Study-Schizophrenia}

We next report an example of methods and preliminary findings for antipsychotic treatment biomarkers in firstepisode SZ from our own mRNA work. We hypothesized that a subset of genes would differentiate and be useful to predict potential responders to two second-generation antipsychotics, olanzapine and risperidone, and test whether gene expression correlates to antipsychotic response to identify responders versus nonresponders prior to treatment.

6.1. Methods. First-episode subjects with schizophrenia were recruited from the inpatient services of the Zucker Hillside Hospital in Glen Oaks, NY, USA and the Bronx-Lebanon Hospital in Bronx, NY, USA as part of a clinical trial in first-episode schizophrenia. Diagnosis of schizophrenia, schizophreniform disorder, or schizoaffective disorder was determined with the Structured Clinical Interview for DSMIV Axis I Disorders (SCID), and subjects were randomly assigned to treatment with olanzapine $(n=15)$ or risperidone $(n=15)$ for 16 weeks. The initial daily dose was $2.5 \mathrm{mg}$ for olanzapine and $1 \mathrm{mg}$ for risperidone. A slowly increasing titration schedule was used: after the first week, dose increases occurred at an interval of one to three weeks until the subject improved or reached a maximum daily dose of $20 \mathrm{mg}$ of olanzapine or six mg of risperidone. Psychopathology was assessed with the Schedule for Affective Disorders and Schizophrenia-Change version with psychosis and disorganized items (SADS-C(+PD)). Response was defined a priori as a rating of mild or better on the (SADS-C $(+\mathrm{PD})$ ) positive symptom items (severity of delusions, severity of hallucinations, impaired understandability, derailment, illogical thinking, and bizarre behavior) plus a CGI rating of much improved or very much improved. The response criteria required that substantial improvement be maintained for two consecutive visits. There were a total of 15 responders and 15 nonresponders (11 males and 4 females per group), taking into account both treatment options (Table 1). When looking at the antipsychotics individually, we have equivalent numbers of responders and nonresponders to treatment (risperidone: $R=8, \mathrm{NR}=8$; olanzapine: $R=7, \mathrm{NR}=$ 7). There was no difference in the numbers of male and females in the responder and nonresponder groups. The age and RIN of the cohort were not statistically different between groups. This cohort was used for both Affymetrix Human Exon 1.0 ST arrays and SYBR Green real-time gene expression assays using the housekeeping gene SLC9A1 as a reference. This cohort was obtained with IRB approval at the Zucker Hillside Hospital. Blood was collected at the onset of the study.

To aid in identification of responders versus nonresponders, we attempted to use previously published gene expression regulation data to identify cis-regulated transcripts associated with treatment response. The mRNA by SNP Browser version 1.0.1 software was used to query the 22 transcripts that had significant "treatment response $\times$ probeset" effect that passed FDR (shown in Table 2) and transcripts with significant "treatment response $\times$ medication $\times$ probeset" effects (See Supplementary Table 1 in Supplementary Material available online at http://dx.doi.org/10.1155/2013/748095). Those transcripts which had significant cis-SNP predictions on expression are shown in Table 4. The mRNA by SNP Browser software [72] contains association results of 54,675 transcripts with 406,912 SNPs $(P<0.05)$ and allows SNPs to be visualized in their genomic context along with linkage disequilibrium maps and putative haplotype blocks derived from the analysis of over 3 million SNPs genotyped in several populations by the International HapMap project.

In a preliminary post hoc analysis of the exon array biomarker expression data, there were 14 subjects who were also genotyped on the Affymetrix $500 \mathrm{~K}$ chip array [73]. We calculated an "SNP $\times$ probeset expression" interaction. Using the results of our screening technique above (Table 4), we then assessed the relationship between genotypes at six SNPs that were identified by the mRNA by SNP Browser or found to be in high linkage disequilibrium to SNPs identified by the mRNA by SNP Browser (see Table 4).

To identify probesets predictive of treatment response, a discriminant analysis was run in Partek Genomics Suite 6.5. A total of 584 variables (all probesets representing the 22 genes with expression significantly associated with treatment response $\times$ probeset) were entered into a forward selection method. The data was divided into two partitions with one level cross validation. Ingenuity Pathway Analysis (Redwood City, CA, USA) was used to determine the canonical pathways to which probesets demonstrating significant $P$ values for "treatment response $\times$ probeset" and "medication $\times$ treatment response $\times$ probeset" interactions belonged. 
TABLE 1: Demographics of first-episode schizophrenia subjects. The mean values \pm standard deviation are shown for RIN, RNA ribosomal band ratio, and age.

\begin{tabular}{lcccccc}
\hline Treatment response & Drug treatment & $N$ & Sex (M/F) & Average RIN & 28S/18S \\
\hline Nonresponder & Olanzapine & 7 & $4 / 3$ & $9.6 \pm 0.3$ & $1.7 \pm 0.2$ & $25.3 \pm 6.3$ \\
Nonresponder & Risperidone & 8 & $7 / 1$ & $9.4 \pm 0.4$ & $1.6 \pm 0.1$ & $25.7 \pm 7.0$ \\
Responder & Olanzapine & 7 & $5 / 2$ & $9.3 \pm 0.6$ & $1.6 \pm 0.1$ & $21.2 \pm 2.1$ \\
Responder & Risperidone & 8 & $6 / 2$ & $9.3 \pm 0.3$ & $1.7 \pm 0.1$ & $22.8 \pm 3.6$ \\
\hline
\end{tabular}

TABLE 2: There were 22 transcripts with statistically significant treatment response $\times$ probeset interaction effects on expression after FDR step-up correction. The Affymetrix transcript ID, gene symbol, nominal $P$ values, FDR step-up $P$ value, and means for each group and largest probeset fold change (FC) are shown in the table. Fold change was calculated as $2^{\text {(responder-nonresponder) }}$.

\begin{tabular}{|c|c|c|c|c|c|c|}
\hline Transcript ID & Gene symbol & $\begin{array}{c}\mathrm{TR} \times \text { probeset } \\
P \text { value }\end{array}$ & $\begin{array}{c}\text { TR } \times \text { probeset } \\
\text { Step-up } \\
P \text { value }\end{array}$ & Mean nonresp. & Mean resp. & $\mathrm{FC}$ \\
\hline 3034987 & ADAP1 & $1.96 \times 10^{-14}$ & $4.24 \times 10^{-10}$ & 1 & 0.86 & 1.3527 \\
\hline 3062794 & TECPR1 & $3.59 \times 10^{-08}$ & $3.19 \times 10^{-04}$ & 0.69 & 0.7 & 1.13796 \\
\hline 2566848 & AFF3 & $4.44 \times 10^{-08}$ & $3.19 \times 10^{-04}$ & -0.09 & 0.08 & -1.52384 \\
\hline 3812922 & NETO1 & $4.08 \times 10^{-07}$ & $2.20 \times 10^{-03}$ & -0.16 & 0.03 & -1.58384 \\
\hline 3521372 & DZIP1 & $5.94 \times 10^{-07}$ & $2.56 \times 10^{-03}$ & -0.32 & -0.46 & 1.44937 \\
\hline 3224650 & DENND1A & $1.40 \times 10^{-06}$ & $5.04 \times 10^{-03}$ & 0.77 & 0.69 & 1.70799 \\
\hline 3933131 & C21orf129 & $1.73 \times 10^{-06}$ & $5.23 \times 10^{-03}$ & -0.1 & -0.09 & -1.45665 \\
\hline 3044283 & CRHR2 & $1.94 \times 10^{-06}$ & $5.23 \times 10^{-03}$ & 0.12 & 0.12 & 1.54165 \\
\hline 2525989 & CPS1 & $2.29 \times 10^{-06}$ & $5.48 \times 10^{-03}$ & -0.43 & -0.28 & -1.30682 \\
\hline 3601229 & $\mathrm{CD} 276$ & $3.33 \times 10^{-06}$ & $7.18 \times 10^{-03}$ & 0.46 & 0.35 & 1.42523 \\
\hline 2773958 & CXCL10 & $4.17 \times 10^{-06}$ & $8.18 \times 10^{-03}$ & -0.56 & -0.31 & -1.54359 \\
\hline 2317317 & TP73 & $4.78 \times 10^{-06}$ & $8.59 \times 10^{-03}$ & 1.14 & 1.02 & 1.24552 \\
\hline 3901296 & CST3 & $5.63 \times 10^{-06}$ & $9.34 \times 10^{-03}$ & 0.93 & 0.83 & 1.31194 \\
\hline 3956781 & AP1B1 & $1.33 \times 10^{-05}$ & 0.02 & 1.45 & 1.37 & 1.19835 \\
\hline 3638760 & IDH2 & $1.46 \times 10^{-05}$ & 0.021 & 1.45 & 1.37 & 1.28415 \\
\hline 3494137 & LMO7 & $1.99 \times 10^{-05}$ & 0.026 & 0.33 & 0.48 & -1.53258 \\
\hline 2450798 & LAD1 & $2.01 \times 10^{-05}$ & 0.026 & 0.57 & 0.51 & 1.2701 \\
\hline 3724858 & TBX21 & $2.22 \times 10^{-05}$ & 0.027 & 0.95 & 0.76 & 1.38771 \\
\hline 3376433 & SLC22A25 & $2.46 \times 10^{-05}$ & 0.028 & -0.58 & -0.58 & -1.16635 \\
\hline 2676182 & NT5DC2 & $2.79 \times 10^{-05}$ & 0.03 & 1.13 & 1.03 & 1.29257 \\
\hline 2930418 & UST & $4.01 \times 10^{-05}$ & 0.041 & 0.24 & 0.58 & -1.56098 \\
\hline 3821847 & ASNA1 & $4.74 \times 10^{-05}$ & 0.046 & 1.48 & 1.43 & 1.32183 \\
\hline
\end{tabular}

TR: treatment response; resp.: responder; nonresp.: nonresponder.

\section{Results}

7.1. Effects of Treatment Response and Medication on Exon Array Expression. The main effects of treatment response (responders versus nonresponders) and medication (olanzapine versus risperidone) on LCL exon expression were examined in first-episode subjects with schizophrenia with no or very limited prior antipsychotic drug treatment. Probeset interaction effects were examined to identify exon-specific changes in expression. Using FDR step-up correction and a significance threshold of 0.05 , there were 22 transcripts with statistically significant treatment response $\times$ probeset effects (Table 2). To identify medication-specific predictors of antipsychotic response, we also examined treatment response $\times$ medication $\times$ probeset effects on expression and identified 245 transcripts passing FDR step-up correction (Supplementary Table 1). Lastly we examined medication effects on expression and identified 210 transcripts with statistically significant medication $\times$ probeset effects (Supplementary Table 2).

7.2. QPCR Validation of Top Ranked Genes. Five candidate genes were selected for qPCR validation based on $P$ value and fold change of probesets with altered expression between responder and nonresponder cell lines. All qPCR results were concordant when compared to microarray in terms of the direction of fold changes. Three of five responder 
TABLE 3: The expression of five treatment response genes and one housekeeping gene was examined by qPCR. Results are shown below. Three genes were significantly altered in responders compared to nonresponders. The fold-change (FC) and $P$ values after normalizing to the housekeeping gene SLC9A1 are shown below. The $\Delta \mathrm{Ct}$ was calculated by subtracting the $\mathrm{Ct}$ of the gene of interest from the $\mathrm{Ct}$ of the housekeeping gene SLC9A1. Fold change was calculated as $2^{\text {-(treatment responder mean - treatment nonresponder mean) }}$ for $\mathrm{qPCR}$ data and $2^{\text {(treatment responder mean - treatment nonresponder mean) }}$ for microarray data. The direction of fold change between diagnosis groups using microarray and qPCR data was consistent for $100 \%$ of the genes. A total of $62 \%$ of attempted validations were significant when gene expression was measured by qPCR.

\begin{tabular}{lcccc}
\hline Gene symbol & Probeset & $P$ value & qPCR FC & Microarray FC \\
\hline NETO & 3812943 & 0.05 & 2.11 & 1.39 \\
AFF3 & 2566939 & 0.05 & 3.25 & 1.35 \\
DENND1A & 3224806 & 0.08 & 0.73 & 0.59 \\
ADAP1 & 3034993 & 0.22 & 0.75 & 0.73 \\
CPS1 & 2526061 & 0.43 & 1.4 & 1.19 \\
\hline
\end{tabular}

TABLE 4: The five most significantly overrepresented canonical pathways identified by Ingenuity Pathway Analysis based on a list of 200 genes with expression associated with treatment response $\times$ probeset or medication $\times$ treatment response $\times$ probeset.

\begin{tabular}{lc}
\hline Treatment response $\times$ probeset $(n=200$ genes $)$ & $\begin{array}{c}\text { B-H } \\
P \text { value }\end{array}$ \\
\hline Axonal guidance signaling & 0.04 \\
Cholecystokinin/gastrin-mediated signaling & 0.22 \\
Role of macrophages, fibroblasts, and endothelial cells & 0.22 \\
in rheumatoid arthritis & 0.26 \\
ERK/MAPK signaling & 0.26 \\
VEGF signaling & B-H \\
\hline Medication $\times$ treatment response $\times$ probeset $(n=200$ & $P$ value \\
genes $)$ & 0.01 \\
\hline A-adrenergic signaling & 0.02 \\
Reelin signaling in neurons & 0.03 \\
Insulin receptor signaling & 0.03 \\
Fc $\gamma$ receptor-medicated phagocytosis in macrophages & \\
and monocytes & 0.03 \\
CREB signaling in neurons &
\end{tabular}

B-H $P$ value: Benjamini-Hochberg false discovery rate.

candidate genes were significantly altered in responders versus nonresponders or showed a trend toward significantly altered expression $(P<0.10)$ (see Table 3$)$.

7.3. Ingenuity Pathway Analysis. Ingenuity Pathway Analysis was conducted on a merged list of the 200 most significant RefSeq transcripts combined with the probesets having the lowest $P$ values for the effects of "treatment response $\times$ probeset" and "medication $\times$ treatment response $\times$ probeset" as the input variables for the data set to query significantly altered named transcripts in responders versus nonresponders (See Table 4 and Supplementary Tables 3 and 4). There

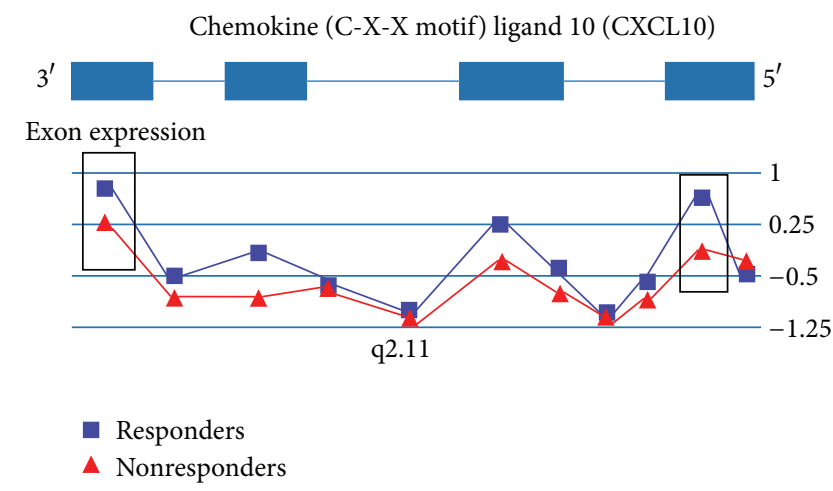

FIGURE 1: This figure depicts a gene view of exon array expression. Treatment responders are shown in blue; nonresponders are shown in red. Median-centered expression values are shown for each probeset within the gene. Treatment responders exhibited a downregulation of CXCL10 relative to treatment nonresponders. We observed a slight effect of SNP rs8878 (Affymetrix probeset SNP_A1871392) genotype on expression of chemokine (C-X-C motif) ligand 10 (CXCL10) probesets 2773961 and 2773970 and treatment response, which are shown in the rectangles. For clarity, error bars representing the standard error of the mean for each probeset were omitted.

was a trend toward an overrepresentation of genes implicated in axonal guidance signaling (12 out of 200 genes, $P=$ 0.04 after Benjamini-Hochberg correction). The 12 genes were ADAM23, ARHGEF11, BMP6, FYN, LINGO1, PRKCD, PTK2, PXN, ROBO1, SEMA4B, VEGFB, and WNT6. The direction of change varied in terms of direction between responders and nonresponders. The remaining most overrepresented pathways are shown in Table 4 .

A second aim of this biomarker study was to identify cisregulated alterations in gene expression using the mRNA by SNP Browser [72] to identify SNP-associated eQTLs (expression quantitative trait loci) for further study in the context of an association with treatment response. The utility of SNPs associated to gene expression as possible biomarkers was previously reviewed by our group [74]. We interrogated the 22 most significant treatment response-associated transcripts, as mentioned above, using the mRNA by SNP Browser. Transcripts with significant eQTLs within the same gene (cisregulated) are listed in Table 5.

In a post hoc analysis of 14 subjects with exon array expression data who were also genotyped on the Affymetrix $500 \mathrm{~K}$ chip array [73], the interaction effect of SNP $\times$ probeset expression was calculated. We observed an association between CXCL10 gene expression and the genotype of rs8878, a $3^{\prime}$-UTR A/G SNP in CXCL10 $(P=0.02)$. We also observed a significant correlation between expression of CXCL10 probesets 2773961 and 2773970 (see Figure 1) and the number of A alleles $(0,1$, or 2$)$.

7.4. Discriminant Analysis. A discriminant analysis was run in Partek Genomics Suite. A total of 584 variables were entered into a forward selection method and analyzed in two partitions. The variables shown to be predictive for each 
TABLE 5: Putative pharmacogenomic candidate SNPs related to treatment response. From the set of 22 named transcripts, 7 genes had significant associations between expression and SNP genotypes according to the mRNA by SNP Browser. Of these 7 genes, the actual expression and genotypes of CXCL10 were associated with treatment response in the 30 subjects measured in this study. When the Illumina array SNP listed in the mRNA SNP Browser was not represented on the Affymetrix array, the nearest Affymetrix SNP chip probeset in high linkage disequilibrium with the Illumina array SNP was identified.

\begin{tabular}{|c|c|c|c|c|c|c|c|}
\hline Gene Symbol & Probeset & Illumina SNP & $P$ value from $m R N A$ by SNP Browser & LOD & Nearest Affymetrix SNP chip probeset & $r^{2}$ & $D^{\prime}$ \\
\hline TAF6 & 203572_s_at & rs13309 & $3.6 \times 10^{-9}$ & 7.6 & SNP_A-2111153 & 0.14 & 0.7 \\
\hline GCLM & 236140_at & rs7515191 & $1.5 \times 10^{-17}$ & 15.8 & SNP_A-1807012 & 0.2 & 1 \\
\hline CCT5 & 229068_at & rs544 & $2.9 \times 10^{-9}$ & 7.7 & SNP_A-1980667 & 1 & 1 \\
\hline ARID3A & 205865_at & rs1051504 & $1.1 \times 10^{-14}$ & 13 & SNP_A-2145232 & 0.18 & 1 \\
\hline CXLC10 & 204533_at & rs8878 & $3.7 \times 10^{-9}$ & 7.5 & SNP_A-1871392 & $*$ & * \\
\hline USP31 & 1558117_s_at & rs10492970 & $1.2 \times 10^{-7}$ & 6.1 & SNP_A-1791706 & * & * \\
\hline GPX7 & 213170_at & rs835342 & $2.0 \times 10^{-18}$ & 16.6 & SNP_A-2236769 & $*$ & $*$ \\
\hline
\end{tabular}

${ }^{*}$ Illumina SNPs and Affymetrix SNPs were identical.

of the partitions were probesets 2526040 (CPS1), 3044290 (CRHR2), 3062804 (TECPR1), and 3812923 (NETO1) for partition one and 3035013 (ADAP1), 2525992 (CPS1), and 3813931 (NETO1) for partition two. Expression of these probesets correctly classified $12 / 15(80 \%)$ of responders and $12 / 15(80 \%)$ of nonresponders. Although this result is encouraging since a cross validation was performed with independent subjects, it warrants confirmation in a larger sample of treated individuals.

\section{Summary of Antipsychotic Treatment Study Findings}

The main finding in this study of SZ antipsychotic treatment response was alteration of 22 transcripts in treatment responders versus nonresponders from subjects with schizophrenia treated with second-generation antipsychotics. The most overrepresented functional group of genes was involved in axonal guidance signaling and included several genes previously linked to schizophrenia. An $80 \%$ cross-validation rate using two sets of subjects was obtained to predict initial responders from nonresponders to antipsychotic treatment during a six-week interval. Although this work requires larger number of subjects and a replication study, it suggests biological pathway differences that influence antipsychotic response leading to successful treatment response. Furthermore, six of the identified transcripts had highly significant cis-regulatory SNPs (see Table 5). From this set of six SNPs, an SNP in the gene CXCL10 was associated with both CXCL10 expression and treatment response. This is of interest due to the associations being found between immune response and neuropsychiatric disorders $[28,68]$ and the association of CXCL10 with antidepressant treatment response in MDD $[28,75,76]$.

\section{Future Directions}

With the advent of next generation sequencing (NGS), clinical researchers have begun to apply this technique to peripheral blood RNA in SZ, MDD, BD, and other disorders
$[77,78]$. The use of NGS can be useful for discovering alternatively spliced RNA, novel long- and short-noncoding RNA, and overall expression levels of RNA that could also be associated with treatment responders. It is envisioned that, once a panel of treatment response biomarkers has been selected and validated, digital tag counting of RNA coding and noncoding molecules from a blood sample could be useful for a clinical test and direct assessment of a panel of biomarkers, eliminating reverse transcription and amplification and improving the reliability of such personalized tests to predict treatment response.

\section{Conclusions}

Major depressive disorder, bipolar disorder, and schizophrenia are devastating disorders affecting at least $15 \%$ of the general population. Many functional genetic candidates have emerged from investigations into the underlying causes of these disorders and treatment response; however, these findings have not been widely replicated. Some likely causal factors for the inconsistency of these biomarker results are the complex nature of psychiatric disorders, as well as individualized treatment response profiles. Complex psychiatric disorders are characterized by an incomplete penetrance, an absence of classic Mendelian transmission likely due to gene $\times$ gene and gene $\times$ environment interactions, genetic heterogeneity, and broad phenotype definitions which translate into decreased power to detect individual gene effects [79]. This complexity is mirrored in treatment outcome, with the majority of subjects not reaping any or limited benefit from treatment. Although much effort has been put toward purely genetic markers of treatment response [2-5], genetic variation alone might not explain response, suggesting that other factors are likely to be involved. This underlies the importance of gene expression studies of response and the need to provide increasing benefit with administered treatments through the identification of robust peripheral biomarkers. The studies reviewed herein provide several accounts of plausible biomarkers of positive treatment response, for example, CXCL10, highlighting several interesting and biological pathways with individual variation such 
as the immune system, while demonstrating the need for larger studies. These further studies are a step towards the identification of biomarkers which can eventually be used in a clinical setting to provide personalized treatment to affected individuals.

\section{Authors' Contribution}

Firoza Mamdani and Maureen V. Martin contributed equally to the paper.

\section{Acknowledgment}

Dr. Vawter is funded by Pritzker Neuropsychiatric Disorders Research Consortium and NIMH MH099440 and MH085801.

\section{References}

[1] C. J. L. Murray and A. D. Lopez, The Global Burden of Disease, WHO, Geneva, Switzerland, 1998.

[2] M. Kato and A. Serretti, "Review and meta-analysis of antidepressant pharmacogenetic findings in major depressive disorder," Molecular Psychiatry, vol. 15, no. 5, pp. 473-500, 2010.

[3] A. K. Malhotra, T. Lencz, C. U. Correll, and J. M. Kane, "Genomics and the future of pharmacotherapy in psychiatry," International Review of Psychiatry, vol. 19, no. 5, pp. 523-530, 2007.

[4] C. Cruceanu, M. Alda, G. Rouleau, and G. Turecki, "Response to treatment in bipolar disorder," Current Opinion in Psychiatry, vol. 24, no. 1, pp. 24-28, 2011.

[5] S. Narasimhan and F. W. Lohoff, "Pharmacogenetics of antidepressant drugs: current clinical practice and future directions," Pharmacogenomics, vol. 13, no. 4, pp. 441-464, 2012.

[6] J.-P. Zhang, T. Lencz, and A. K. Malhotra, "D2 receptor genetic variation and clinical response to antipsychotic drug treatment: a meta-analysis," American Journal of Psychiatry, vol. 167, no. 7, pp. 763-772, 2010.

[7] A. Bromberg, Y. Bersudsky, J. Levine, and G. Agam, "Global leukocyte DNA methylation is not altered in euthymic bipolar patients," Journal of Affective Disorders, vol. 118, no. 1-3, pp. 234239, 2009.

[8] A. Carrard, A. Salzmann, A. Malafosse, and F. Karege, "Increased DNA methylation status of the serotonin receptor 5HTR1A gene promoter in schizophrenia and bipolar disorder," Journal of Affective Disorders, vol. 132, no. 3, pp. 450-453, 2011.

[9] Z. Kaminsky, M. Tochigi, P. Jia et al., "A multi-tissue analysis identifies HLA complex group 9 gene methylation differences in bipolar disorder," Molecular Psychiatry, vol. 17, pp. 728-740, 2012.

[10] M. Kinoshita, S. Numata, A. Tajima et al., "DNA methylation signatures of peripheral leukocytes in schizophrenia," NeuroMolecular Medicine, vol. 15, no. 1, pp. 95-101, 2013.

[11] A. Menke, T. Klengel, and E. B. Binder, "Epigenetics, depression and antidepressant treatment," Current Pharmaceutical Design, vol. 18, no. 36, pp. 5879-5889, 2012.

[12] M. Schroeder, M. O. Krebs, S. Bleich, and H. Frieling, "Epigenetics and depression: current challenges and new therapeutic options," Current Opinion in Psychiatry, vol. 23, no. 6, pp. 588592, 2010.
[13] V. Vialou, J. Feng, A. J. Robison, and E. J. Nestler, "Epigenetic mechanisms of depression and antidepressant action," Annual Review of Pharmacology and Toxicology, vol. 53, pp. 59-87, 2013.

[14] J. P. Lopez, F. Mamdani, B. Labonte et al., "Epigenetic regulation of BDNF expression according to antidepressant response," Molecular Psychiatry, vol. 18, pp. 398-399, 2013.

[15] Y. Dwivedi, "Brain-derived neurotrophic factor: role in depression and suicide," Neuropsychiatric Disease and Treatment, vol. 5, no. 1, pp. 433-449, 2009.

[16] M. L. Molendijk, B. A. A. Bus, P. Spinhoven et al., "Serum levels of brain-derived neurotrophic factor in major depressive disorder: state-trait issues, clinical features and pharmacological treatment," Molecular Psychiatry, vol. 16, no. 11, pp. 1088-1095, 2011.

[17] J. C. Chang, E. C. Wooten, A. Tsimelzon et al., "Gene expression profiling for the prediction of therapeutic response to docetaxel in patients with breast cancer," The Lancet, vol. 362, no. 9381, pp. 362-369, 2003.

[18] K. Nagasaki and Y. Miki, "Molecular prediction of the therapeutic response to neoadjuvant chemotherapy in breast cancer," Breast Cancer, vol. 15, no. 2, pp. 117-120, 2008.

[19] H. Zembutsu, Y. Suzuki, A. Sasaki et al., "Predicting response to docetaxel neoadjuvant chemotherapy for advanced breast cancers through genome-wide gene expression profiling," International Journal of Oncology, vol. 34, no. 2, pp. 361-370, 2009.

[20] A. J. Schetter, S. Y. Leung, J. J. Sohn et al., "MicroRNA expression profiles associated with prognosis and therapeutic outcome in colon adenocarcinoma," Journal of the American Medical Association, vol. 299, no. 4, pp. 425-436, 2008.

[21] M. M. Kittleson, K. M. Minhas, R. A. Irizarry et al., "Gene expression in giant cell myocarditis: altered expression of immune response genes," International Journal of Cardiology, vol. 102, no. 2, pp. 333-340, 2005.

[22] N. Marziliano, M. Grasso, A. Pilotto et al., "Transcriptomic and proteomic analysis in the cardiovascular setting: unravelling the disease?" Journal of Cardiovascular Medicine, vol. 10, no. 5, pp. 433-442, 2009.

[23] S. J. Matkovich, D. J. van Booven, K. A. Youker et al., "Reciprocal regulation of myocardial microRNAs and messenger RNA in human cardiomyopathy and reversal of the microRNA signature by biomechanical support," Circulation, vol. 119, no. 9, pp. 1263-1271, 2009.

[24] R. V. Saveanu and C. B. Nemeroff, "Etiology of depression: genetic and environmental factors," Psychiatric Clinics of North America, vol. 35, no. 1, pp. 51-71, 2012.

[25] B. Rollins, M. V. Martin, L. Morgan, and M. P. Vawter, "Analysis of whole genome biomarker expression in blood and brain," American Journal of Medical Genetics B, vol. 153, no. 4, pp. 919936, 2010.

[26] C.-C. Liew, J. Ma, H.-C. Tang, R. Zheng, and A. A. Dempsey, "The peripheral blood transcriptome dynamically reflects system wide biology: a potential diagnostic tool," Journal of Laboratory and Clinical Medicine, vol. 147, no. 3, pp. 126-132, 2006.

[27] P. F. Sullivan, C. Fan, and C. M. Perou, "Evaluating the comparability of gene expression in blood and brain," American Journal of Medical Genetics B, vol. 141, no. 3, pp. 261-268, 2006.

[28] P. A. Sequeira, M. V. Martin, and M. P. Vawter, “The first decade and beyond of transcriptional profiling in schizophrenia," Neurobiology of Disease, vol. 45, no. 1, pp. 23-36, 2012. 
[29] M. P. Vawter, E. Ferran, B. Galke, K. Cooper, W. E. Bunney, and W. Byerley, "Microarray screening of lymphocyte gene expression differences in a multiplex schizophrenia pedigree," Schizophrenia Research, vol. 67, no. 1, pp. 41-52, 2004.

[30] M. T. Tsuang, N. Nossova, T. Yager et al., "Assessing the validity of blood-based gene expression profiles for the classification of schizophrenia and bipolar disorder: a preliminary report," American Journal of Medical Genetics B, vol. 133, no. 1, pp. 1-5, 2005.

[31] S. J. Glatt, I. P. Everall, W. S. Kremen et al., "Comparative gene expression analysis of blood and brain provides concurrent validation of SELENBP1 up-regulation in schizophrenia," Proceedings of the National Academy of Sciences of the United States of America, vol. 102, no. 43, pp. 15533-15538, 2005.

[32] F. A. Middleton, C. N. Pato, K. L. Gentile et al., "Gene expression analysis of peripheral blood leukocytes from discordant sib-pairs with schizophrenia and bipolar disorder reveals points of convergence between genetic and functional genomic approaches," American Journal of Medical Genetics B, vol. 136, no. 1, pp. 12-25, 2005.

[33] E. McNeill and V. D. van, "MicroRNAs shape the neuronal landscape," Neuron, vol. 75, pp. 363-379, 2012.

[34] E. Gardiner, N. J. Beveridge, J. Q. Wu et al., "Imprinted DLK1-DIO3 region of 14q32 defines a schizophrenia-associated miRNA signature in peripheral blood mononuclear cells," Molecular Psychiatry, vol. 17, pp. 827-840, 2012.

[35] C.-Y. Lai, S.-L. Yu, M. H. Hsieh et al., "MicroRNA expression Aberration as potential peripheral blood biomarkers for Schizophrenia," PLoS One, vol. 6, no. 6, Article ID e21635, 2011.

[36] H. Chen, N. Wang, M. Burmeister, and M. G. McInnis, "MicroRNA expression changes in lymphoblastoid cell lines in response to lithium treatment," International Journal of Neuropsychopharmacology, vol. 12, no. 7, pp. 975-981, 2009.

[37] R. Zhou, P. Yuan, Y. Wang et al., "Evidence for selective microRNAs and their effectors as common long-term targets for the actions of mood stabilizers," Neuropsychopharmacology, vol. 34, no. 6, pp. 1395-1405, 2009.

[38] L. Bocchio-Chiavetto, E. Maffioletti, P. Bettinsoli et al., "Blood microRNA changes in depressed patients during antidepressant treatment," European Neuropsychopharmacology, 2012.

[39] K. Oved, A. Morag, M. Pasmanik-Chor et al., "Genome-wide miRNA expression profiling of human lymphoblastoid cell lines identifies tentative SSRI antidepressant response biomarkers," Pharmacogenomics, vol. 13, pp. 1129-1139, 2012.

[40] A. Baudry, S. Mouillet-Richard, B. Schneider, J.-M. Launay, and O. Kellermann, "MiR-16 targets the serotonin transporter: a new facet for adaptive responses to antidepressants," Science, vol. 329, no. 5998, pp. 1537-1541, 2010.

[41] T. Matsubara, H. Funato, A. Kobayashi, M. Nobumoto, and Y. Watanabe, "Reduced glucocorticoid receptor $\alpha$ expression in mood disorder patients and first-degree relatives," Biological Psychiatry, vol. 59, no. 8, pp. 689-695, 2006.

[42] M. Fujimoto, S. Uchida, T. Watanuki et al., "Reduced expression of glyoxalase-1 mRNA in mood disorder patients," Neuroscience Letters, vol. 438, no. 2, pp. 196-199, 2008.

[43] K. Otsuki, S. Uchida, T. Watanuki et al., "Altered expression of neurotrophic factors in patients with major depression," Journal of Psychiatric Research, vol. 42, no. 14, pp. 1145-1153, 2008.

[44] J.-I. Iga, S.-I. Ueno, K. Yamauchi et al., "Gene expression and association analysis of LIM (PDLIM5) in major depression," Neuroscience Letters, vol. 400, no. 3, pp. 203-207, 2006.
[45] J.-I. Iga, S.-I. Ueno, K. Yamauchi et al., "Altered HDAC5 and CREB mRNA expressions in the peripheral leukocytes of major depression," Progress in Neuro-Psychopharmacology and Biological Psychiatry, vol. 31, no. 3, pp. 628-632, 2007.

[46] J.-I. Iga, S.-I. Ueno, K. Yamauchi et al., "Serotonin transporter mRNA expression in peripheral leukocytes of patients with major depression before and after treatment with paroxetine," Neuroscience Letters, vol. 389, no. 1, pp. 12-16, 2005.

[47] C.-W. Tsao, Y.-S. Lin, C.-C. Chen, C.-H. Bai, and S.-R. Wu, "Cytokines and serotonin transporter in patients with major depression," Progress in Neuro-Psychopharmacology and Biological Psychiatry, vol. 30, no. 5, pp. 899-905, 2006.

[48] L. Lima, S. Mata, and M. Urbina, "Allelic isoforms and decrease in serotonin transporter mRNA in lymphocytes of patients with major depression," NeuroImmunoModulation, vol. 12, no. 5, pp. 299-306, 2005.

[49] R. H. Segman, T. Goltser-Dubner, I. Weiner et al., "Blood mononuclear cell gene expression signature of postpartum depression," Molecular Psychiatry, vol. 15, no. 1, pp. 93-100, 2010.

[50] S. Spijker, J. S. van Zanten, S. de Jong et al., "Stimulated gene expression profiles as a blood marker of major depressive disorder," Biological Psychiatry, vol. 68, no. 2, pp. 179-186, 2010.

[51] A. Reichenberg, R. Yirmiya, A. Schuld et al., "Cytokineassociated emotional and cognitive disturbances in humans," Archives of General Psychiatry, vol. 58, no. 5, pp. 445-452, 2001.

[52] S. M. Kurian, H. Le-Niculescu, S. D. Patel et al., "Identification of blood biomarkers for psychosis using convergent functional genomics," Molecular Psychiatry, vol. 16, no. 1, pp. 37-58, 2011.

[53] M. Takahashi, H. Hayashi, Y. Watanabe et al., "Diagnostic classification of schizophrenia by neural network analysis of bloodbased gene expression signatures," Schizophrenia Research, vol. 119, no. 1-3, pp. 210-218, 2010.

[54] N. A. Bowden, J. Weidenhofer, R. J. Scott et al., "Preliminary investigation of gene expression profiles in peripheral blood lymphocytes in schizophrenia," Schizophrenia Research, vol. 82, no. 2-3, pp. 175-183, 2006.

[55] M. R. Kuzman, V. Medved, J. Terzic, and D. Krainc, "Genomewide expression analysis of peripheral blood identifies candidate biomarkers for schizophrenia," Journal of Psychiatric Research, vol. 43, no. 13, pp. 1073-1077, 2009.

[56] M. Maschietto, A. R. Silva, R. D. Puga et al., "Gene expression of peripheral blood lymphocytes may discriminate patients with schizophrenia from controls," Psychiatry Research, vol. 200, pp. 1018-1021, 2012.

[57] T. Hobara, S. Uchida, K. Otsuki et al., "Altered gene expression of histone deacetylases in mood disorder patients," Journal of Psychiatric Research, vol. 44, no. 5, pp. 263-270, 2010.

[58] M. Ventriglia, R. Zanardini, L. Pedrini et al., "VEGF serum levels in depressed patients during SSRI antidepressant treatment," Progress in Neuro-Psychopharmacology and Biological Psychiatry, vol. 33, no. 1, pp. 146-149, 2009.

[59] R. Belzeaux, C. Formisano-Tréziny, A. Loundou et al., "Clinical variations modulate patterns of gene expression and define blood biomarkers in major depression," Journal of Psychiatric Research, vol. 44, no. 16, pp. 1205-1213, 2010.

[60] R. Belzeaux, A. Bergon, V. Jeanjean et al., "Responder and nonresponder patients exhibit different peripheral transcriptional signatures during major depressive episode," Translational Psychiatry, vol. 2, p. e185, 2012.

[61] F. Mamdani, M. T. Berlim, M.-M. Beaulieu, A. Labbe, C. Merette, and G. Turecki, "Gene expression biomarkers of 
response to citalopram treatment in major depressive disorder," Translational Psychiatry, vol. 1, article el3, 2011.

[62] A. O. Vik-Mo, A. B. Birkenaes, J. Fernø, H. Jonsdottir, O. A. Andreassen, and V. M. Steen, "Increased expression of lipid biosynthesis genes in peripheral blood cells of olanzapinetreated patients," International Journal of Neuropsychopharmacology, vol. 11, no. 5, pp. 679-684, 2008.

[63] J. S. De, M. P. Boks, T. F. Fuller et al., "A gene co-expression network in whole blood of schizophrenia patients is independent of antipsychotic-use and enriched for brain-expressed genes," PLoS One, vol. 7, Article ID e39498, 2012.

[64] P. Jia, L. Wang, A. H. Fanous, X. Chen, K. S. Kendler, and Z. Zhao, "A bias-reducing pathway enrichment analysis of genome-wide association data confirmed association of the MHC region with schizophrenia," Journal of Medical Genetics, vol. 49, no. 2, pp. 96-103, 2012.

[65] M. Debnath, D. M. Cannon, and G. Venkatasubramanian, "Variation in the major histocompatibility complex [MHC] gene family in schizophrenia: associations and functional implications," Progress in Neuro-Psychopharmacology \& Biological Psychiatry, vol. 42, pp. 49-62, 2013.

[66] A. Gutiérrez-Fernández, A. González-Pinto, P. Vega, S. Barbeito, and C. Matute, "Expression of oligodendrocyte and myelin genes is not altered in peripheral blood cells of patients with first-episode schizophrenia and bipolar disorder," Bipolar Disorders, vol. 12, no. 1, pp. 107-109, 2010.

[67] R. D. Beech, L. Lowthert, J. J. Leffert et al., "Increased peripheral blood expression of electron transport chain genes in bipolar depression," Bipolar Disorders, vol. 12, no. 8, pp. 813-824, 2010.

[68] M. A. Zain, S. N. Jahan, G. P. Reynolds, N. Z. Zainal, S. Kanagasundram, and Z. Mohamed, "Peripheral PDLIM5 expression in bipolar disorder and the effect of olanzapine administration," BMC Medical Genetics, vol. 13, p. 91, 2012.

[69] K. Kikuchi, J.-I. Iga, S. Tayoshi et al., "Lithium decreases VEGF mRNA expression in leukocytes of healthy subjects and patients with bipolar disorder," Human Psychopharmacology, vol. 26, no. 4-5, pp. 358-363, 2011.

[70] H. Sugawara, K. Iwamoto, M. Bundo et al., "Effect of mood stabilizers on gene expression in lymphoblastoid cells," Journal of Neural Transmission, vol. 117, no. 2, pp. 155-164, 2010.

[71] L. Lowthert, J. Leffert, A. Lin et al., "Increased ratio of anti-apoptotic to pro-apoptotic Bcl2 gene-family members in lithium-responders one month after treatment initiation," Biology of Mood \& Anxiety Disorders, vol. 2, p. 15, 2012.

[72] F. M. de la Vega, "Selecting single-nucleotide polymorphisms for association studies with SNPbrowserTM software," Methods in Molecular Biology, vol. 376, pp. 177-193, 2007.

[73] P. DeRosse, T. Lencz, K. E. Burdick, S. G. Siris, J. M. Kane, and A. K. Malhotra, "The genetics of symptom-based phenotypes: toward a molecular classification of schizophrenia," Schizophrenia Bulletin, vol. 34, no. 6, pp. 1047-1053, 2008.

[74] M. P. Vawter, F. Mamdani, and F. Macciardi, "An integrative functional genomics approach for discovering biomarkers in Schizophrenia," Briefings in Functional Genomics, vol. 10, no. 6, pp. 387-399, 2011.

[75] M. Michel, M. J. Schmidt, and K. Mirnics, "Immune system gene dysregulation in autism and schizophrenia," Developmental Neurobiology, vol. 72, pp. 1277-1287, 2012.

[76] M.-L. Wong, C. Dong, J. Maestre-Mesa, and J. Licinio, "Polymorphisms in inflammation-related genes are associated with susceptibility to major depression and antidepressant response," Molecular Psychiatry, vol. 13, no. 8, pp. 800-812, 2008.
[77] J. Sainz, I. Mata, J. Barrera et al., "Inflammatory and immune response genes have significantly altered expression in schizophrenia," Molecular Psychiatry, 2012.

[78] A. Sequeira, M. V. Martin, B. Rollins et al., "Mitochondrial mutations and polymorphisms in psychiatric disorders," Frontiers in Genetics, vol. 3, p. 103, 2012.

[79] E. S. Lander and N. J. Schork, "Genetic dissection of complex traits," Science, vol. 265, no. 5181, pp. 2037-2048, 1994. 


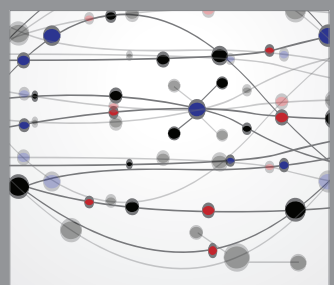

The Scientific World Journal
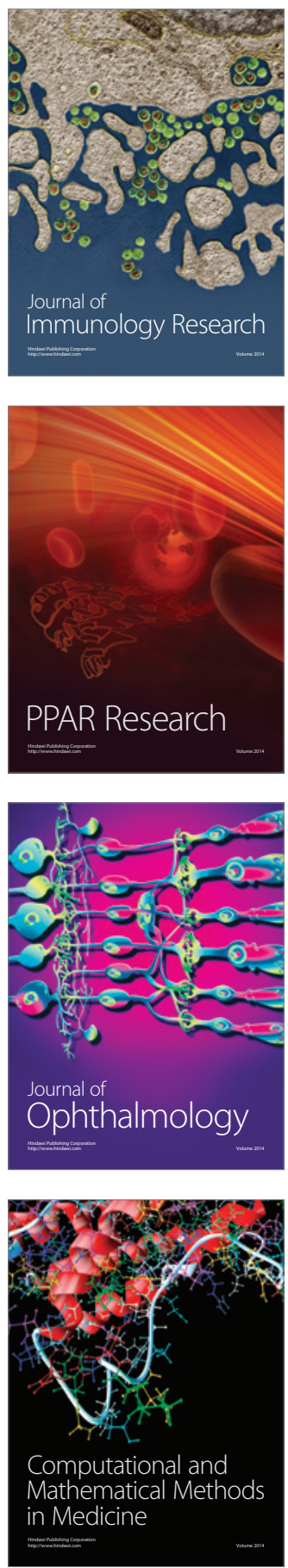

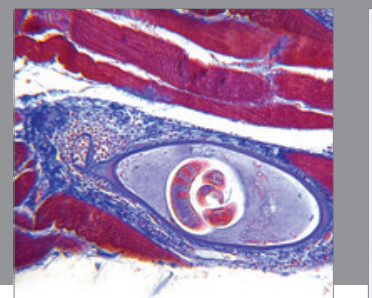

Gastroenterology

Research and Practice
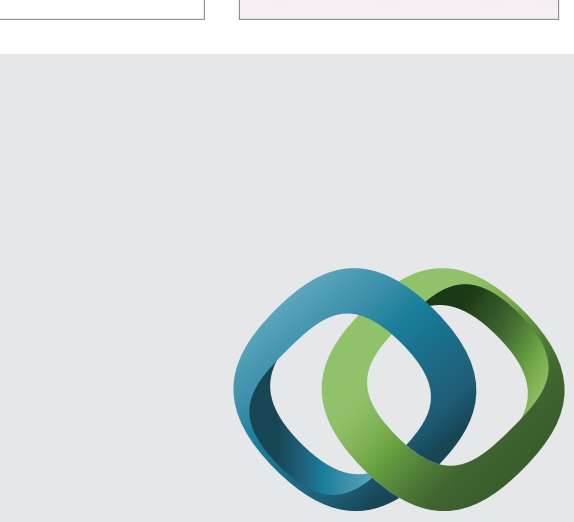

\section{Hindawi}

Submit your manuscripts at

http://www.hindawi.com
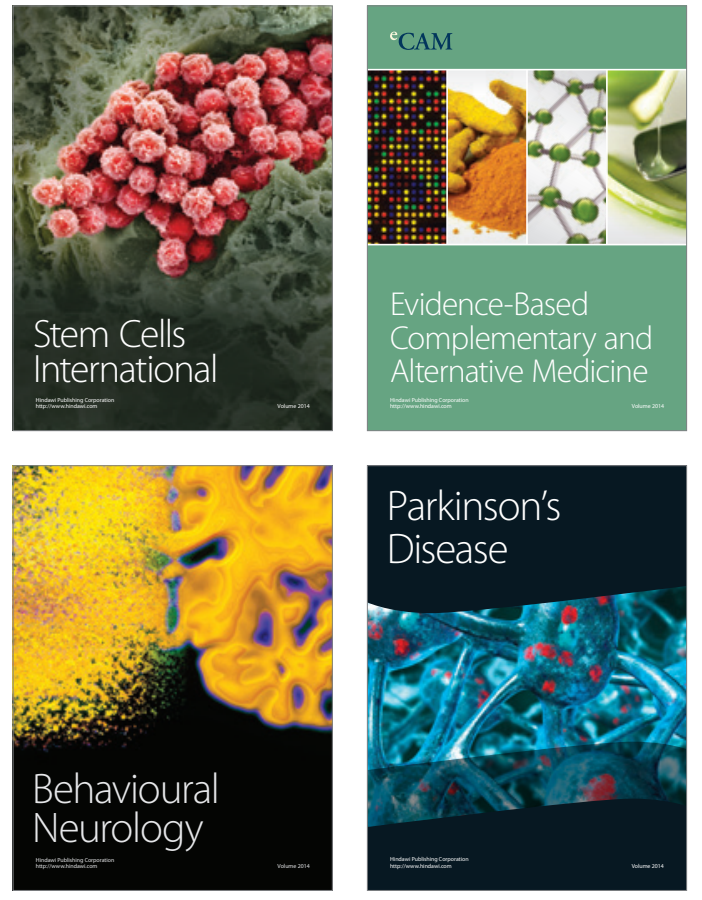
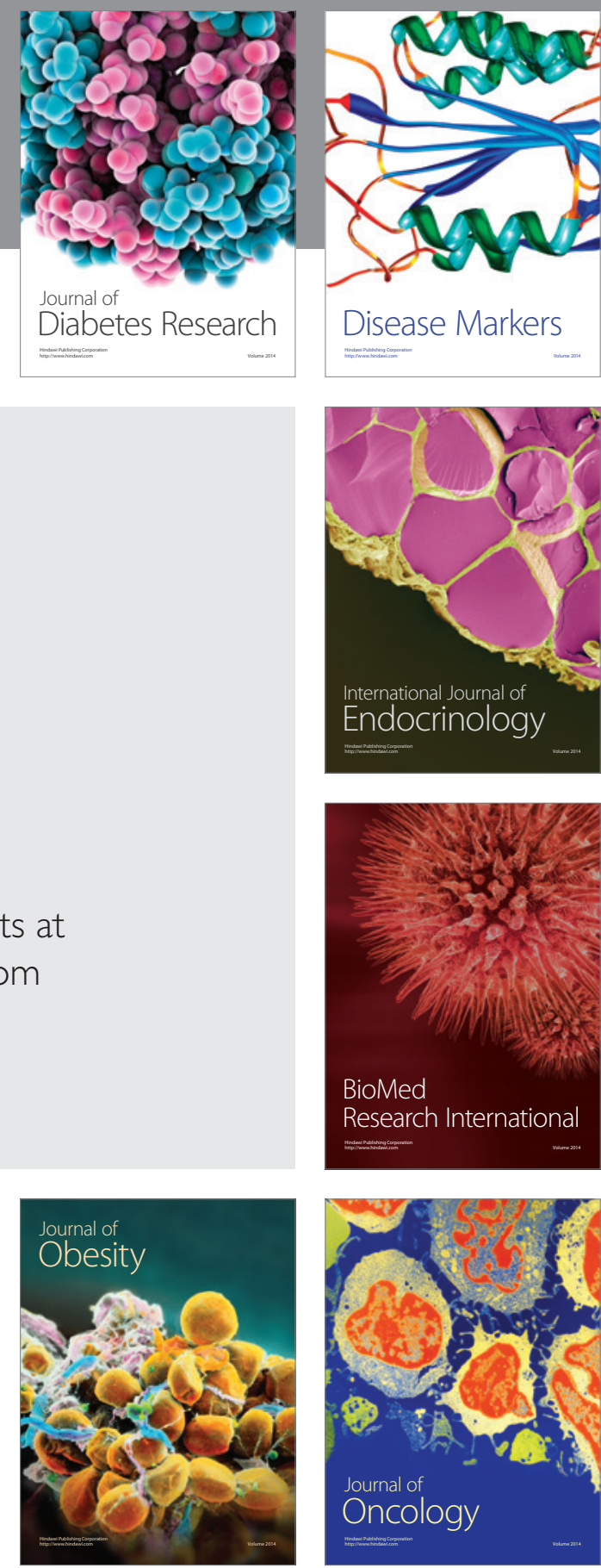

Disease Markers
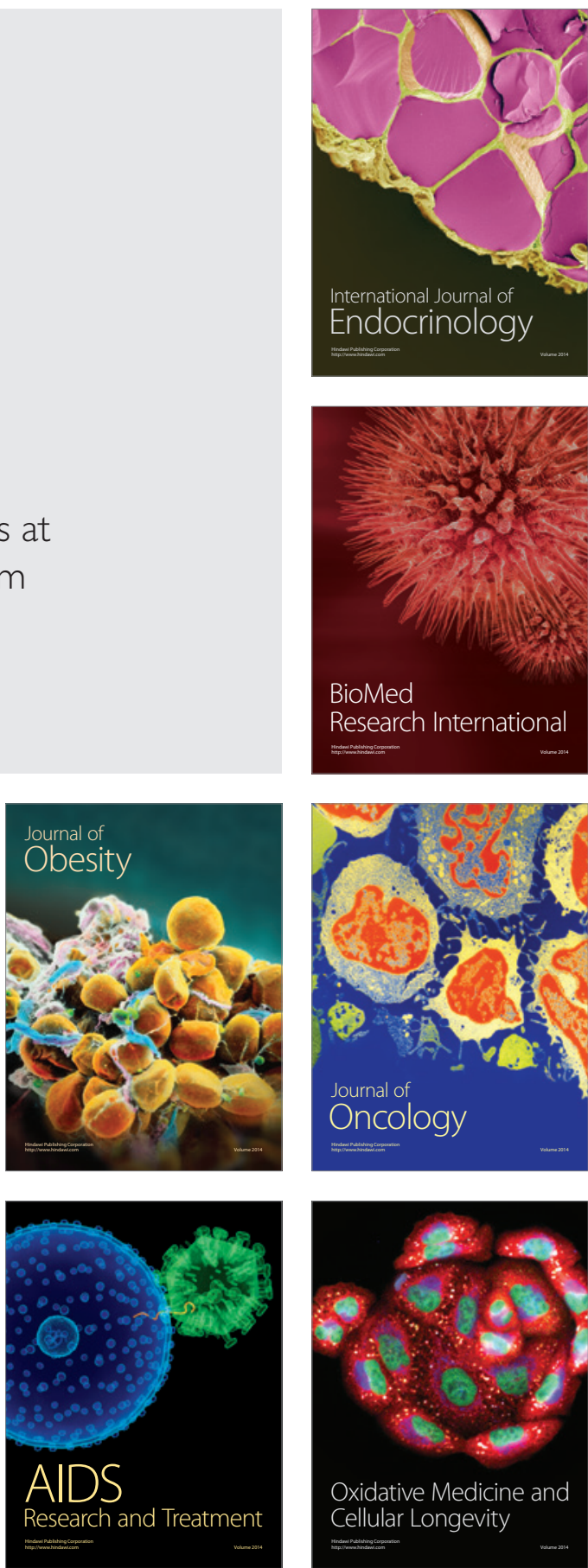